\title{
Progress, challenges, and future perspectives in genetic researches of stuttering
}

\author{
Changsoo Kang*๑ \\ Department of Biotechnology and Institute of Basic Sciences, Sungshin Women's University, Seoul, Korea
}

\begin{abstract}
Speech and language functions are highly cognitive and human-specific features. The underlying causes of normal speech and language function are believed to reside in the human brain. Developmental persistent stuttering, a speech and language disorder, has been regarded as the most challenging disorder in determining genetic causes because of the high percentage of spontaneous recovery in stutters. This mysterious characteristic hinders speech pathologists from discriminating recovered stutters from completely normal individuals. Over the last several decades, several genetic approaches have been used to identify the genetic causes of stuttering, and remarkable progress has been made in genome-wide linkage analysis followed by gene sequencing. So far, four genes, namely GNPTAB, GNPTG, NAGPA, and AP4E1, are known to cause stuttering. Furthermore, thegeneration of mouse models of stuttering and morphometry analysis has created new ways for researchers to identify brain regions that participate in human speech function and to understand the neuropathology of stuttering. In this review, we aimed to investigate previous progress, challenges, and future perspectives in understanding the genetics and neuropathology underlying persistent developmental stuttering.
\end{abstract}

Key words: Speech, Stuttering, Lysosome, Genetics.

\section{Introduction}

Most animals communicate with each other in diverse ways using sounds, gestures, and facial movements to warn about danger, claim territory, and seek attraction from the opposite sex [1]. Compared to non-human species, human communication is mediated by proper sounds and remarkably complex verbal and grammatical pledges in a systematic manner, which helps them to dominate other species on Earth [2,3]. Human-specific communication comprises of speech and language, which are often difficult to distinguish between them. Speech is often defined as mechanical sounds of spoken language and includes articu- lations, which are the way sounds are generated. In contrast, language is a word and a combined sequence of words that constitute meaningful phrases or sentences based on accepted grammatical rules [4].

Although disruptions in the function of communication skills are not mostly life-threatening in humans, affected individuals have significant difficulties in leading a normal life. Speech and language disorders include developmental verbal dyspraxia, dyslexia, specific language impairment, and stuttering. Stuttering, also called stammering, is regarded as the most common speech disorder. It is well characterized by repetitions, prolongation of syllables, or by unintended halts in the sequential flow of

\footnotetext{
Received: 15 November 2021, Revised: 11 December 2021, Accepted: 13 December 2021, Published: 31 December 2021

*Corresponding author: Changsoo Kang, Ph.D. (iD https://orcid.org/0000-0003-1854-9491

Department of Biotechnology and Institute of Basic Sciences, Sungshin Women's University, 55 Dobong-ro 76ga-gil, Gangbuk-gu, Seoul 01133, Korea.

Tel: +82-2-920-7638, Fax: +82-0504-381-7638, E-mail: ckang@sungshin.ac.kr

Conflict of interest: I declare that I do not have any conflicts of interest.

(c) This is an open-access article distributed under the terms of the Creative Commons Attribution Non-Commercial License (http://creativecommons.org/licenses/by-nc/4.0/) which permits unrestricted non-commercial use, distribution, and reproduction in any medium, provided the original work is properly cited.

(c) Copyright 2021 by the Korean Society of Medical Genetics and Genomics 
the speech known as blocks [4]. Clinical diagnosis is performed by measuring the four aspects of speech behaviors including frequency, sustainment time, physical accompanying features, and fluency of the individual's speech using a stuttering severity instrument-4 [5]. This disorder may be accompanied by eye blinks, lip tremors, facial tics, and clenching fists. Similar to other speech disorders, stuttering occurs in young children with a typical onset age of approximately 3 years, with a male-tofemale prevalence rate of 2:1. While it affects approximately $5 \%$ of the population, independent of the affected individual's ethnicity or spoken language, the majority of the stutters (80\%) resolve spontaneously or with the help of clinical speech therapy [6]. This recovery is observed more commonly in female than in male stutters, thereby increasing the male-to-female prevalence rate to $4: 1$ by the age of nine. The overall prevalence rate of persistent stuttering is approximately $1 \%$ in the general population.

Several genetic studies have been conducted to identify causative genes and their roles in speech and language disorders, including stuttering. Recently, evidence has accumulated to support genetic contributions that increase the susceptibility to stuttering. Here, we review past significant genetic discoveries and functional studies to reveal the causes of stuttering (Table 1).

\section{Evidence of Genetic Contributions to Stuttering}

Like several other disorders, it was suggested that stuttering is attributed to both non-genetic and genetic factors; thus, the extent to which the risk of this disorder was attributable to these two factors was an initial task that needed to be resolved. There are a few genetic approaches to address this challenge.

First, adoption studies of stuttering are an appropriate method to evaluate the influence of nature and nurture on this disorder. These studies investigated adopted stutters in multiple families, and the results were inconclusive because the sample size was not large enough to indicate statistical significance [7]. Another study group recruited 156 adopted and non-adopted children, and it was determined that the affection risk in adopted children with a genetic background of stuttering was higher than that in adopted children with no known genetic background [8]. In addition, it was reported that stuttering may not be acquired by persistent communication with parents affected by speech disorders. These two adoption studies indicated that genetic factors, rather than family environment, are better predictors of the affection status of the offspring $[7,8]$. Second, together with the adoption studies aforementioned,

Table 1. Notable studies contributed to understand genetic causes of stuttering

\begin{tabular}{|c|c|c|c|}
\hline Study group [reference] & Year & Method & Major finding \\
\hline Shugart et al. [18] & 2004 & $\begin{array}{l}\text { Genome-wide linkage scans in } 68 \text { North American } \\
\text { stuttering families }\end{array}$ & $\begin{array}{l}\text { Found non-parametric linkage score of } 5.35 \text { at the } \\
\text { marker on chromosome } 18 p\end{array}$ \\
\hline Riaz et al. [26] & 2005 & $\begin{array}{l}\text { Genome-wide linkage analysis in Pakistani } \\
\text { consanguineous families }\end{array}$ & $\begin{array}{l}\text { Found significant genetic linkage to markers on the } \\
\text { chromosome } 12 q\end{array}$ \\
\hline Wittke-Thompson et al. [20] & 2007 & $\begin{array}{l}\text { Genome-wide linkage and association analyses in } \\
\text { a large founder population named Hutterites }\end{array}$ & $\begin{array}{l}\text { Identified suggestive linkage to the microsatellite } \\
\text { markers on chromosomes } 2 \text { and } 5\end{array}$ \\
\hline Kang et al. [27] & 2010 & $\begin{array}{l}\text { Sanger Sequencing of linkage region of chromosome } \\
12 q\end{array}$ & $\begin{array}{l}\text { Found association of stuttering with mutations in the } \\
\text { GNPTAB, GNPTG and NAGPA genes }\end{array}$ \\
\hline Lee et al. [31] & 2011 & $\begin{array}{l}\text { Enzyme assay for the mutations found in the GNPTAB } \\
\text { and NAGPA }\end{array}$ & $\begin{array}{l}\text { Demonstrated that the mutations in the GNPTAB and } \\
\text { NAGPA result in partial loss of activities }\end{array}$ \\
\hline Fedyna et al. [28] & 2011 & $\begin{array}{l}\text { Haplotype analysis with the SNP markers surrounding } \\
\text { GNPTAB p.Glu1200Lys mutation }\end{array}$ & $\begin{array}{l}\text { Revealed that the variant of GNPTAB p.Glu1200Lys is } \\
\text { a founder mutation generated approximately } \\
14,300 \text { years ago }\end{array}$ \\
\hline Raza et al. [32] & 2013 & $\begin{array}{l}\text { Whole-genome linkage analysis using SNP chips and } \\
\text { microsatellite markers in the large west African } \\
\text { stuttering family }\end{array}$ & $\begin{array}{l}\text { Detected significant LOD scores ranging from } \\
4.7-6.6 \text { on the chromoses } 2 p \text { and } 15 q\end{array}$ \\
\hline Raza et al. [33] & 2015 & $\begin{array}{l}\text { Whole-exome sequencing of Cameroonian stuttering } \\
\text { family }\end{array}$ & $\begin{array}{l}\text { Suggested association of the mutations in AP4E1 } \\
\text { gene with persistent stuttering }\end{array}$ \\
\hline Barnes et al. [36] & 2016 & $\begin{array}{l}\text { Analysis of ultrasonic vocalizations in mouse model of } \\
\text { stuttering }\end{array}$ & $\begin{array}{l}\text { Found altered vocalization patterns in the knock-in } \\
\text { mouse carrying Gpnmb mutation. }\end{array}$ \\
\hline Han et al. [37] & 2019 & $\begin{array}{l}\text { Characterized mouse model of stuttering with mutations } \\
\text { in the Gnptab gene }\end{array}$ & $\begin{array}{l}\text { Detected altered ultrasonic vocalization in the } \\
\text { knock-in mouse and found astrocyte deficits in the } \\
\text { corpus callosum }\end{array}$ \\
\hline Choo et al. [40] & 2011 & $\begin{array}{l}\text { Analyzed gene expression data at Allen Brain Institute } \\
\text { and voxel-based morphometry }\end{array}$ & $\begin{array}{l}\text { Association of lysosomal enzyme trafficking genes } \\
\text { with area size of gray matter in stutters and normal } \\
\text { subjects }\end{array}$ \\
\hline
\end{tabular}

SNP, single-nucleotide polymorphism; LOD, logarithm of odds ratio. 
twin studies are another useful approach for evaluating genetic contributions to diseases. Several genetic investigations of dizygotic and monozygotic twin pairs with at least one stutter were performed to estimate heritability and mode of inheritance in stuttering [9-13]. It was reported that the concordance rate for this disorder was much higher in identical twins (63\%) than in the fraternal twins (19\%), suggesting that genetic factors contribute to the etiology of stuttering. However, the possibility that environmental factors have an effect on stuttering could not be completely excluded as several of the monozygotic twins in the study were discordant for stuttering, which implies that both non-genetic and genetic factors contribute to the etiology of stuttering [11]. While these adoption and twin studies varied in sample size, ethnicity, diagnostic criteria, and statistical methods, the results consistently supported the evidence of genetic influences on stuttering. The overall heritability estimates from these studies ranged from 0.42 to 0.85 [13-15]. Third, Cox et al. $[16,17]$ ascertained modestly sized stuttering families with multiple individuals affected by stuttering. With no findings on the involvement of non-genetic factors such as anxiety levels, familial attitudes toward speech, and ratings of parental behavior, the ascertainment of family clustering of stuttering was also strong evidence suggesting a genetic contribution to stuttering.

\section{Genome-wide Linkage Scans and Association Studies}

Genetic studies in the twins and adoptees, coupled with the finding of family clustering of stutters, implied the contribution of genetics contributions to the susceptibility to stuttering. These results developed a few study groups to perform parametric or non-parametric genome-wide linkage analyses to identify genetic loci and further mutated genes in the families of European descent ascertained in North America $[18,19]$. These two studies showed only suggestive linkage to chromosomes $2 q, 9 p$, $15 q, 18 p$, and 18q, while another study ascertained an isolated population, called Hutterrite, but the results from neither multipoint linkage analysis nor meta-analysis met the genome-wide statistical criteria [20].

In addition to genome-wide linkage analysis in families, association studies in the unrelated case-control groups might be an alternative approach in the genetic studies of stuttering. A few association studies were performed in the Kurdish and Han Chinese populations, and it was reported that two singlenucleotide polymorphisms (SNPs) in each of the cytochrome
P450 family 17 (CYP17) and dopamine D2 receptor (DRD2) were nominally associated with susceptibility to stuttering $[21,22]$; however, these suggestive associations were not replicated in the other two case-control studies in more than 1,500 Caucasian and Brazilian individuals [23,24].

These failures in family-based genome-wide linkage and casecontrol replication studies may be ascribed to the two reasons. First, the transmission of stuttering in the populations does not follow the typical Mendelian mode of inheritance; therefore, the highly complex segregation pattern made it difficult to perform appropriate statistical analyses. Second, stuttering resolves spontaneously in most of the affected family members (>70\%), especially females; therefore, there is a chance that unaffected individuals might have recovered from their former stuttering status. This raises the question of whether the clinical diagnosis regards recovered stutters as affected or not. Thus, these puzzling features of stuttering have hindered successful genetic studies of stuttering for several decades.

In genetic studies, the employment of consanguineous families is often advantageous and may increase the success of identifying causative genes owing to the homogeneous genomic structure in the family. Consanguineous marriages in the families usually create a pedigree structure with a significantly increased incidence of recessive genetic disorders than in outbreeding families. In addition, when their genome is sequenced, the number of candidate variants found is dramatically decreased because of the high homogeneity of the genomic variants in these families, allowing researchers to readily pinpoint causative mutations readily [25]. Thus, the same is likely true for complex disorders such as stuttering. From this perspective, genetic studies in the Pakistani stuttering familiy by Riaz et al. [26] could be promising because $60 \%$ to $70 \%$ of all matings were between cousins in the family. The authors ascertained 46 highly inbred families from the city of Lahore, Pakistan, and significant genetic linkage (nonparametric logarithm of odds ratio [LOD] score $=4.6$ ) was detected in microsatellite variants dispersed on the chromosome 12q. These strong linkage results were attributed to the enrichment of consanguineous marriages in the recruited families.

\section{GNPTAB Gene and Developmental Persistent Stuttering}

The first causative gene for stuttering was discovered by a research group led by Dr. Drayna at the National Institute on Deafness and Other Communication Disorders, National Insti- 


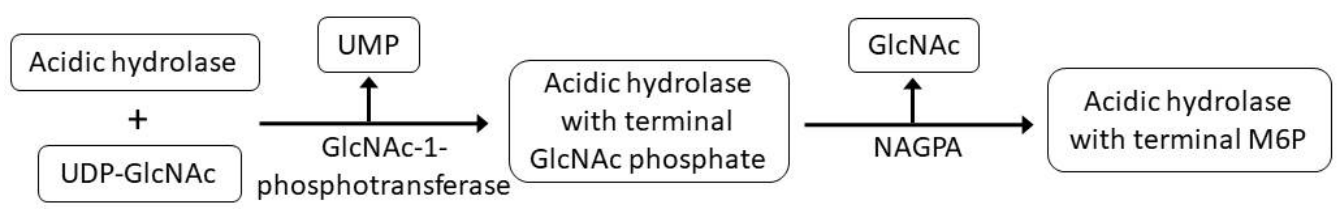

Fig. 1. Synthesis of M6P determinants on the acidic hydrolases in the trans-Golgi network. GIcNAc-1-phosphotransferase transfer GIcNAcphosphate moiety from UDP-GIcNAc to the oligosaccharides of acidic hydrolases. NAGPA uncovers M6P on the oligosaccharides by cleaving off terminal GIcNAc. UDP-GIcNAc, uridine diphosphate N-acetylglucosamine; UMP, uridine monophosphate; NAGPA, N-acetylglucosamine-1-phosphodiester alpha-N-acetylglucosaminidase; M6P, mannose 6-phosphate.

tutes of Health, USA. They focused on the largest family, designated PKST72, out of 46 families that had previously undergone genome-wide linkage scans [26]. Upon further bioinformatic investigation of the University of California, Santa Cruz (UCSC) genome database (https://genome.ucsc.edu), they identified that there were 87 genes within the $10 \mathrm{Mb}$ linkage region on the chromosome 12q23.3 bounded by D12S101 and D12S1597 microsatellite markers were identified. Sequencing exons, exon/intron junctions and promoter regions in these 87 genes revealed that a mutation (c.3598G>A, p.Glu1200Lys, rs137853825) in the GNPTAB gene (NM_024312, Online Mendelian Inheritance in Man [OMIM] \#607840) was most highly segregated together with the stuttering phenotype in the PKST72 family [27]. This missense mutation resulted in the substitution of normal glutamate with lysine at the amino acid position of 1,200 in the GNPTAB gene, and was found in three other Pakistani stuttering families. The presence of this mutation in 4 out of 46 Pakistani families implied that the genetic contribution of this mutation to stuttering might account for $8.7 \%$ of the Pakistani population.

Subsequent haplotype analysis of the $650 \mathrm{~kb}$ region surrounding the GNPTAB c.3598G>A (p.Glu1200Lys, rs137853825) site in the individuals carrying this mutation revealed that this mutation arose not by repeated mutation at this position but by sharing a founder haplotype generated about 14,300 years ( 572 generations) ago [28].

\section{Lysosomal Enzyme-trafficking Pathway and Developmental Persistent Stuttering}

The GNPTAB and GNPTG genes encode two alpha, two beta, and two gamma subunits of the $\mathrm{N}$-acetylglucosamine-1-phosphotransferase (GIcNAc-1-phosphotransferase, EC 2.7.8.17), respectively [29]. This hexameric enzyme catalyzes the transfer of a GICNAc-phosphate moiety from uridine diphosphateGlcNAc onto the N-linked high mannose oligosaccharide of the lysosomal hydrolases, which is the first step in the synthesis of a mannose 6-phosphate (M6P) signal tag onto the newly synthesized acidic hydrolases destined to lysosomes from the Golgi complex (Fig. 1) [29]. The second step in generating M6P signals on the glycans of lysosomal enzymes is mediated by $\mathrm{N}$-acetylglucosamine-1-phosphodiester alpha- $\mathrm{N}$-acetylglucosaminidase (NAGPA), also known as uncovering enzyme (EC 3.1.4.45), which removes the terminal GlcNAc moiety, thereby uncovering M6P, which is recognized by M6P receptors in the Golgi complex (Fig. 1) [30].

Considering that both GICNAc-1-phosphotransferase and NAGPA enzymes are involved in the two-step process of attaching the M6P tagging signal onto the lysosomal hydrolases as described above, it could be rationally hypothesized that genetic mutations in the GNPTG (OMIM \#607838) and NAGPA (OMIM \#607985) might result in the same phenotypic effect as that induced by the GNPTAB gene. Additional genetic studies of functionally related GNPTG and NAGPA genes, and their robust sequencing of them in the 393 unrelated stutters with Pakistani or European ancestry revealed three mutations, which were not found in the 372 neurologically normal controls [27]. Thus, in 25 out of 393 (6.4\%) unrelated stuttering individuals, genetic differences were ascribed to failures in the transportation of the acidic hydrolases from the trans-Golgi network to the lysosome.

Data on the molecular mechanisms underlying how genetic mutations in the GNPTAB and GNPTG contribute to the occurrence of stuttering are not yet available; however, the functional consequences of the two NAGPA mutations (p.Arg328Cys and p.Phe513SerfsX113) have been reported [31]. In an enzyme assay to estimate the conversion of M6P diesters to monoesters, the mutant NAGPA expressing p.Arg328Cys (rs139526942) or p.Phe 513SerfsX113 mutation exhibited solely $37 \%$ conversion efficiency which was significantly lower than the $51 \%$ conversion by normal cells $(P$-value $=0.01)$. Other data revealing the effect of the genetic mutations on the intracellular localization of the NAGPA were obtained by treating both wild-type and mutant NAGPA with or without endo $\mathrm{Hf}$ or peptide N-glycosidase (PNGase) F. The majority of the wild-type NAGPAs were resistant 
to endo $\mathrm{Hf}$ but sensitive to PNGase $\mathrm{F}$, which implied that they were mostly localized in the trans-Golgi network. In contrast, the mutant NAGPAs harboring p.Arg328Cys or p.Phe513SerfsX113 were sensitive to both endo $\mathrm{Hf}$ and $\mathrm{PNG}$ Gase F treatment. In addition, pulse-chase experiments using ${ }^{35} \mathrm{~S}-\mathrm{Met} / \mathrm{Cys}$ radioisotopes on NAGPA, were performed to compare the half-lives of the wild-type and mutants, and it was shown that the half-life of the wild-type NAGPA was much longer (14 hours) than that of p.Arg328Cys (7 hours) and p.Phe513SerfsX113 ( $<1$ hour) mutants. Thus, these functional assays mentioned above support the hypothesis that if folded properly, newly translated normal NAGPA escape the endoplasmic reticulum (ER), and readily translocate into the Golgi complex; however, mutant NAGPAs are caught in the ER for longer periods and are vulnerable to ER-associated degradation machinery thereby resulting in only 50\% of them trafficking to the Golgi [31].

Although genetic mutations in the NAGPA have been shown to disrupt it's normal trafficking to the Golgi complex, it is totally unknown how the NAGPA mutations result in persistent stuttering which presumed to arise from neurological dysfunction in the human brain.

\section{AP4E1 and Developmental Persistent Stuttering}

Other remarkable genetic studies of stuttering have investigated a large Cameroonian family harboring 33 individuals affected by developmental persistent stuttering. Raza et al. [32] performed genome-wide linkage scans by combining genotyping 332 microsatellites (Marshifield Weber 10 panel) and 6,090 SNPs (Human Linkage 12 Panel). Initial analyses of the entire family found no genetic markers associated with stuttering. The authors then divided the large family into five subfamilies, and finally discovered significant linkage in the sub-family named $1 \mathrm{E}$ to the markers on chromosomes $2 p$ and $15 q$ with LOD scores ranging from 4.7 to 6.6. Further whole-exome sequencing of these linkage regions and subsequent bioinformatic analyses in the Cameroonian subfamily members discovered two heterozygous mutations (p.Val517lle [rs7600211635] and p.Glu801 Lys [rs556450190]) in the AP4E1 gene (OMIM \# 184450), which cosegregated with a stuttering phenotype. The overall genetic contribution of this gene to the occurrence of scattering was estimated to be $2.1 \%$ to $3.7 \%$ of the persistent unrelated stutters [33]. The AP4E1 gene encodes the epsilon-1 subunit of the adaptor-related protein complex 4 , which is also known to be involved in protein trafficking in the trans-Golgi complex by directly interacting with NAGPA [34]. This functional link between
AP4E1 and NAGPA supports the evidence that trafficking of acidic hydrolases from the trans-Golgi network to the lysosomes is associated with persistent stuttering.

\section{Mouse Model of Stuttering and Ultrasonic Vocalization}

Animal models are useful tools for the genetic studies of human diseases. In this point of view, generating a mouse model of speech and language disorders is a major challenge because speech and language are human-specific functions. Interestingly, it was discovered that mice communicate with each other by generating ultrasonic vocalizations at a frequency of 30 to $110 \mathrm{kHz}$ [35]. Based on this finding, a knock-in mouse model of stuttering was engineered to determine whether the mutations found in the genes of lysosomal enzyme-trafficking pathway genes lead to the same or similar phenotypes found in from human stutters. This study found out that mice carrying mutations in the Gnptab gene generated fewer ultrasonic vocalizations and had significantly longer pauses between syllables compared with wild-type littermates. Thus, these altered vocalizations in mutant mice mimicked typical characteristics of human stuttering; therefore, stuttering mouse models might be a promising animal tool for further understanding of the underlying mechanisms of this disorder [36].

While the mouse model of stuttering displayed abnormal features similar to human stutters, the location and role of the speech center, which is assumed to be in the brain, are entirely unknown. Han et al. [37] cleverly introduced Gnptab Ser321Gly and Ala455Ser mutations into mice by using a cell type-specific Cre-drivers and conditional knock-out tools, and reported that these mice showed altered ultrasonic vocalizations but normal in non-vocal behaviors. In addition, they found that only the astrocyte-specific Gnptab knockout mice showed an abnormal vocalization pattern, which raised the hypothesis that brain astrocytes, particularly in the corpus callosum, may be one of the potential brain regions where speech neurons may reside.

\section{Future Challenges and Perspectives}

For several decades, many studies have been performed to identify the genetic causes of stuttering, and to date a few genes, particularly those associated with intracellular protein trafficking pathways, are known to cause this human-specific disorder. Although links between these genes, namely the GNPT- 
$A B, G N P T G, N A G P A$, and $A P 4 E 1$ genes to stuttering are convincing, the overall genetic contributions of these four lysosomal enzyme-trafficking pathway genes to stuttering account for only $10 \%$ of the developmental persistent stuttering individuals, which implies that the majority of the stuttering individuals might be ascribed to the deficits in other genes. Considering previous genome-wide linkage data, additional genes associated with stuttering may reside within the chromosomes $2 p, 3 q$, $14 q$, and $21 q$ chromosomes, which harbor markers linked with stuttering.

Mutations in the GNPTAB, GNPTG, and AP4E1 genes were originally found in patients with mucolipidosis II (OMIM \#252650), mucolipidosis III (OMIM \#252605), and spastic paraplegia 51 (OMIM \#607244), respectively. These are metabolic disorders, and they follow a typical recessive mode of inheritance, almost all of which eliminate the activities of the proteins encoded by these genes; however, most mutations found in stutters are missense, small insertional, or deleterious, which leads to the partial loss of the protein activity. Thus, this is one of the examples of a genetic study in which different mutations in the same genes may cause different disorders. The underlying mechanisms of how this complete or partial losses of function results in different disorders are totally unknown to date, and it remains to be investigated in the future.

Unlike GNPTAB, GNPTG, and AP4E1, mutations in the NAGPA gene have not been associated with human disorders other than persistent developmental stutters; therefore, further investigation of the neurological function of this gene might be a starting point in the study to reveal the molecular mechanisms underlying speech function in the human brain. It is also noteworthy that the human NAGPA gene has several isoforms, one of which is solely expressed in the human brain [30]. In addition, this isoform is found in humans, but not in the mouse brain. Thus, the fact of human brain-specific expression of this NAGPA isoform leads to the hypothesis that this isoform may play an important role in the brain region participating in speech function in humans. Before testing this hypothesis, the sub-brain region called the speech center should be determined.

For several decades, many studies have been performed to pinpoint speech centers in the human brain, and it has been suggested that the Broca's and the Wernicke's areas are the brain regions responsible for speech and language functions $[38,39]$. In addition, the corpus callosum, which is involved in interhemispheric processing by interconnecting the left and right hemispheres of the brain may be another potential brain region for human speech, because it was reported that the overall area of the corpus callosum was significantly larger in the stutters than in normal individuals [40]. However, the detailed association between corpus callosum size and stuttering at the cellular level is currently unknown.

\section{Conclusion}

For the last several decades, tremendous effort has been devoted to discovering the genetic causes associated with speech and language disorders, including stuttering. Although, the enigmatic characteristics of the stuttering, such as a high spontaneous recovery rate, have hindered the success of these studies, employment of consanguineous Pakistani, and large Cameroonian stuttering families have enabled researchers to identify genetic mutations in the GNPTAB, GNPTG, NAGPA, and $A P 4 E 1$ as the genetic causes of stuttering. All of these genes are involved in the cellular transportation of acidic hydrolases destined from the trans-Golgi network to the lysosomes. Partial loss of function in the enzymes encoded by these genes accounts for approximately $2.1 \%$ to $3.7 \%$ of the developmental persistent stutters, worldwide. Therefore, most of the genetic architecture of stuttering is still unknown. To identify more stuttering associated genes and to better understand the neuropathology of persistent stuttering, new next-generation sequencing technologies for the transcriptome, exome and genome in the single cells derived from potential speech neurons need to be exploited in future studies.

\section{Acknowledgements}

This work was supported by the National Research Foundation of Korea funded by the Korean Government (Ministry of Science and ICT) (Grant no. 2021R1F1A1058330).

\section{References}

1. Snyder PJ. The evolution of communication: an ethological exploration. Brain Lang 1998;62:465-8.

2. Petitto LA, Marentette PF. Babbling in the manual mode: evidence for the ontogeny of language. Science 1991;251:1493-6.

3. Fisher SE, Marcus GF. The eloquent ape: genes, brains and the evolution of language. Nat Rev Genet 2006;7:9-20.

4. Bloodstein O, Ratner NB, Brundage SB. A handbook on stuttering. 7th ed. San Diego (CA): Plural Publishing; 2021. 581 p.

5. Riley GD. A stuttering severity instrument for children and adults. J Speech Hear Disord 1972;37:314-22. 
6. Smith A, Weber C. How stuttering develops: the multifactorial dynamic pathways theory. J Speech Lang Hear Res 2017;60:2483-505.

7. Bloodstein O. Stuttering in families of adopted stutterers. J Speech Hear Disord 1961;26:395-6.

8. Felsenfeld S, Plomin R. Epidemiological and offspring analyses of developmental speech disorders using data from the Colorado Adoption Project. J Speech Lang Hear Res 1997;40:778-91.

9. Felsenfeld S, Kirk KM, Zhu G, Statham DJ, Neale MC, Martin NG. A study of the genetic and environmental etiology of stuttering in a selected twin sample. Behav Genet 2000;30:359-66.

10. Andrews G, Morris-Yates A, Howie P, Martin NG. Genetic factors in stuttering confirmed. Arch Gen Psychiatry 1991;48:1034-5.

11. Howie PM. Concordance for stuttering in monozygotic and dizygotic twin pairs. J Speech Hear Res 1981;24:317-21.

12. Rautakoski P, Hannus T, Simberg S, Sandnabba NK, Santtila P. Genetic and environmental effects on stuttering: a twin study from Finland. J Fluency Disord 2012;37:202-10.

13. Fagnani $C_{1}$ Fibiger $S$, Skytthe $A$, Hjelmborg JV. Heritability and environmental effects for self-reported periods with stuttering: a twin study from Denmark. Logoped Phoniatr Vocol 2011;36:114-20.

14. van Beijsterveldt CE, Felsenfeld S, Boomsma DI. Bivariate genetic analyses of stuttering and nonfluency in a large sample of 5-yearold twins. J Speech Lang Hear Res 2010;53:609-19.

15. Ooki S. Genetic and environmental influences on stuttering and tics in Japanese twin children. Twin Res Hum Genet 2005;8:69-75.

16. Cox NJ, Kramer PL, Kidd KK. Segregation analyses of stuttering. Genet Epidemiol 1984;1:245-53.

17. Cox NJ, Seider RA, Kidd KK. Some environmental factors and hypotheses for stuttering in families with several stutterers. J Speech Hear Res 1984;27:543-8.

18. Shugart YY, Mundorff J, Kilshaw J, Doheny K, Doan B, Wanyee J, et al. Results of a genome-wide linkage scan for stuttering. Am J Med Genet A 2004;124A:133-5.

19. Suresh $\mathrm{R}$, Ambrose $\mathrm{N}$, Roe $\mathrm{C}$, Pluzhnikov A, Wittke-Thompson JK, Ng $M C_{1}$ et al. New complexities in the genetics of stuttering: significant sex-specific linkage signals. Am J Hum Genet 2006;78:554-63.

20. Wittke-Thompson JK, Ambrose N, Yairi E, Roe C, Cook EH, Ober C, et al. Genetic studies of stuttering in a founder population. J Fluency Disord 2007;32:33-50.

21. Lan J, Song M, Pan C, Zhuang G, Wang Y, Ma W, et al. Association between dopaminergic genes (SLC6A3 and DRD2) and stuttering among Han Chinese. J Hum Genet 2009;54:457-60.

22. Mohammadi $H_{1}$ Joghataei MT, Rahimi $Z$, Faghihi $F_{1}$ Khazaie $H_{1}$ Farhangdoost $H_{\text {, et }}$ al. Sex steroid hormones and sex hormone binding globulin levels, CYP17 MSP Al (-34T:C) and CYP19 codon 39 (Trp:Arg) variants in children with developmental stuttering. Brain Lang
2017;175:47-56.

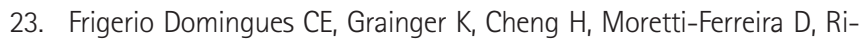
azuddin S, Drayna D. Are variants in sex hormone metabolizing genes associated with stuttering? Brain Lang 2019;191:28-30.

24. Kang C, Domingues BS, Sainz E, Domingues CE, Drayna D, MorettiFerreira D. Evaluation of the association between polymorphisms at the DRD2 locus and stuttering. J Hum Genet 2011;56:472-3.

25. Hamamy H. Consanguineous marriages: preconception consultation in primary health care settings. J Community Genet 2012;3:185-92.

26. Riaz N, Steinberg S, Ahmad J, Pluzhnikov A, Riazuddin S, Cox NJ, et al. Genomewide significant linkage to stuttering on chromosome 12 Am J Hum Genet 2005;76:647-51.

27. Kang C, Riazuddin S, Mundorff J, Krasnewich D, Friedman P, Mullikin $\mathrm{JC}$, et al. Mutations in the lysosomal enzyme-targeting pathway and persistent stuttering. N Engl J Med 2010;362:677-85.

28. Fedyna A, Drayna D, Kang C. Characterization of a mutation commonly associated with persistent stuttering: evidence for a founder mutation. J Hum Genet 2011;56:80-2.

29. Kollmann K, Pohl S, Marschner K, Encarnação M, Sakwa I, Tiede S, et al. Mannose phosphorylation in health and disease. Eur J Cell Biol 2010;89:117-23.

30. Kornfeld R, Bao M, Brewer K, Noll C, Canfield W. Molecular cloning and functional expression of two splice forms of human $\mathrm{N}$-acetylglucosamine-1-phosphodiester alpha-N-acetylglucosaminidase. J Biol Chem 1999;274:32778-85.

31. Lee WS, Kang C, Drayna D, Kornfeld S. Analysis of mannose 6-phosphate uncovering enzyme mutations associated with persistent stuttering. J Biol Chem 2011;286:39786-93.

32. Raza MH, Gertz EM, Mundorff J, Lukong J, Kuster J, Schäffer AA, et al Linkage analysis of a large African family segregating stuttering suggests polygenic inheritance. Hum Genet 2013;132:385-96.

33. Raza MH, Mattera $R$, Morell $R$, Sainz $E$, Rahn $R$, Gutierrez J, et al. Association between rare variants in AP4E1, a component of intracellular trafficking, and persistent stuttering. Am J Hum Genet 2015;97:715-25.

34. Dell'Angelica EC, Mullins C, Bonifacino JS. AP-4, a novel protein complex related to clathrin adaptors. J Biol Chem 1999;274:7278-85.

35. Holy TE, Guo Z. Ultrasonic songs of male mice. PLoS Biol 2005;3:e386.

36. Barnes TD, Wozniak DF, Gutierrez J, Han TU, Drayna D, Holy TE. A mutation associated with stuttering alters mouse pup ultrasonic vocalizations. Curr Biol 2016;26:1009-18.

37. Han TU, Root J, Reyes LD, Huchinson EB, Hoffmann JD, Lee WS, et al. Human GNPTAB stuttering mutations engineered into mice cause vocalization deficits and astrocyte pathology in the corpus callosum. Proc Natl Acad Sci U S A 2019;116:17515-24.

38. Damasio AR. Aphasia. N Engl J Med 1992;326:531-9. 
39. Poeppel D, Hickok G. Towards a new functional anatomy of language. Cognition 2004;92:1-12.

40. Choo AL, Kraft SJ, Olivero W, Ambrose NG, Sharma H, Chang SE, et al.
Corpus callosum differences associated with persistent stuttering in adults. J Commun Disord 2011;44:470-7. 